\title{
Public health information and statistics dissemination efforts for Indonesia on the Internet
}

\author{
Febiana Hanani ${ }^{1}$, Takashi Kobayashi ${ }^{1}$, Eitetsu Jo $^{1}$, Sawako Nakajima ${ }^{1}$, Hiroshi Oyama ${ }^{1}$ \\ ${ }^{1}$ Department of Clinical Information Engineering, Health Services Sciences, School of \\ Public Health, Graduate School of Medicine, The University of Tokyo, Japan
}

\begin{abstract}
Objectives: To elucidate current issues related to health statistics dissemination efforts on the Internet in Indonesia and to propose a new dissemination website as a solution.
\end{abstract}

Methods: A cross-sectional survey was conducted. Sources of statistics were identified using link relationship and Google ${ }^{\mathrm{TM}}$ search. Menu used to locate statistics, mode of presentation and means of access to statistics, and available statistics were assessed for each site. Assessment results were used to derive design specification; a prototype system was developed and evaluated with usability test.

Results: 49 sources were identified on 18 governmental, 8 international and 5 non-government websites. Of 49 menus identified, 33\% used non-intuitive titles and lead to inefficient search. $69 \%$ of them were on government websites. Of 31 websites, only $39 \%$ and $23 \%$ used graph/chart and map for presentation. Further, only 32\%, 39\% and 19\% provided query, export and print feature. While $>50 \%$ sources reported morbidity, risk factor and service provision statistics, $<40 \%$ sources reported health resource and mortality statistics. Statistics portal website was developed using Joomla! ${ }^{\mathrm{TM}}$ content management system. Usability test demonstrated its potential to improve data accessibility.

Discussion and conclusion: In this study, government's efforts to disseminate statistics in Indonesia are supported by non-governmental and international organizations and existing their information may not be very useful because it is: a) not widely distributed, b) difficult to locate, and c) not effectively communicated. Actions are needed to ensure information usability, and one of such actions is the development of statistics portal website.

Keywords: public health, public information, statistics, web services, usability 


\section{Introduction}

Indonesia has achieved significant improvement in health over the past decades as indicated by the decrease in infant mortality rate and the prolonged life expectancy [[1]]. New challenges, however, have emerged as a result of health transition, economic development, globalization and decentralization [[1] [2]]. Epidemiological transition, i.e. double burden of disease, imposes a huge pressure on health program and system in Indonesia [[1] [2]]. Economic development and globalization contribute to the spread of major risk factors for non-communicable diseases such as tobacco and obesity [[1] [2]]. Further, decentralization, under which local governments are given larger autonomy, poses challenge for them on how to manage their new autonomy so as to benefit local community [[1]].

The three core functions of public health, assessment, policy development and assurance, as outlined in The Future of Public Health [[3]], emphasize the significance of health statistics for public health decision-making. Health statistics are needed to assess population health, plan, implement and evaluate the effectiveness of health program, and allocate health resources [[4]]. Nowadays, statistics that include a wider array of health issues from communicable diseases and maternal/child health to non-communicable diseases, disaggregated by socioeconomic and demographic characteristics, are needed to develop effective responses to existing challenges [[5]].

The Government of Indonesia in its National Health Information System (NHIS) Policy and Strategy has acknowledged that the provision of accurate, updated and timely presentation of information is a prerequisite for the achievement of healthy Indonesia [[6]]. One of the NHIS's missions is the provision of information for various purposes and different information users [[6]]. Government's efforts to provide health statistics for the public have been joined by other public bodies such as international organizations. To date, however, no effort has been made to identify how far along are they in their efforts especially in which they are using websites as dissemination tools.

In contrast to healthcare information, there have been few studies of health statistics on the web. Raban et al. reviewed the availability of health statistics for India [[7]] while ORC Macro surveyed US Government's sites used for statistics dissemination [[8]]. 


\section{Objectives}

Thus, the objectives of this research are to elucidate current issues related to health statistics dissemination efforts on the Internet in Indonesia, and to propose a solution when the need arises.

\section{Methods}

Our review methodology was based on those used in Raban's study[[7]]. The following steps were done subsequently: 1) identification of sources of health statistics available on the web, 2) assessment of menu used to locate statistics on website, 3) assessment of mode of presentation and means of access to statistics, 4) assessment of available statistics, and 5) whenever appropriate, offer solution to issue identified in the assessment.

Review of the characteristics of websites offering health statistics for Indonesia:

1) Identification of sources of health statistics available on the web

Standard search using search engine would be inadequate for this purpose because many of Indonesian websites were not indexed or indexed with keyword that could not represent the contents of the sites. Having identified government websites well-known for providing statistics (Ministry of Health, Central Bureau of Statistics and Directorate General of Population Administration) and using the knowledge that government sites often provide links to other sites with related content, we opted to search on websites linked to the well-known sites, and on any link on these websites (Figure 1-a).

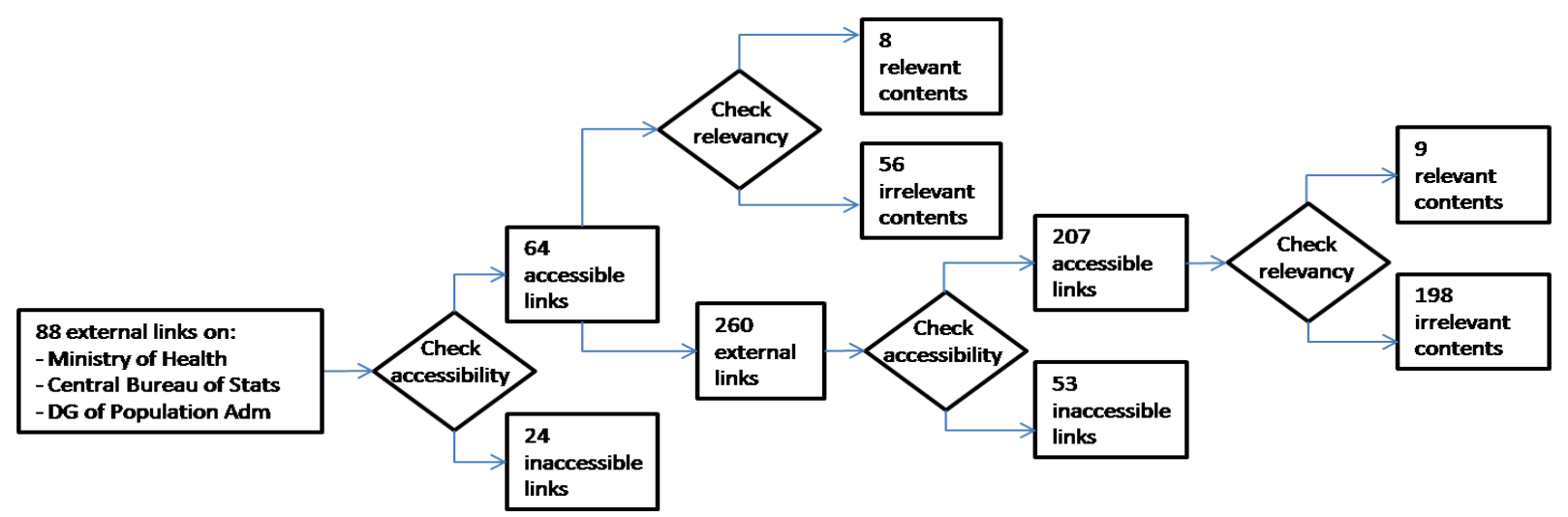

Figure 1-a. The diagram of search results of public health statistics websites using link relationship

We focused on sites belong to the Government, non-governmental and international organizations as they are reliable sources of information. Another reason why we did not use 
search engine as our first strategy was because it was difficult to choose the appropriate search keywords. The only keyword that we identified as quite specific and yet not too broad was "health survey" and thus, we used it to identify all surveys that we might have missed on our first search (Figure 1-b).

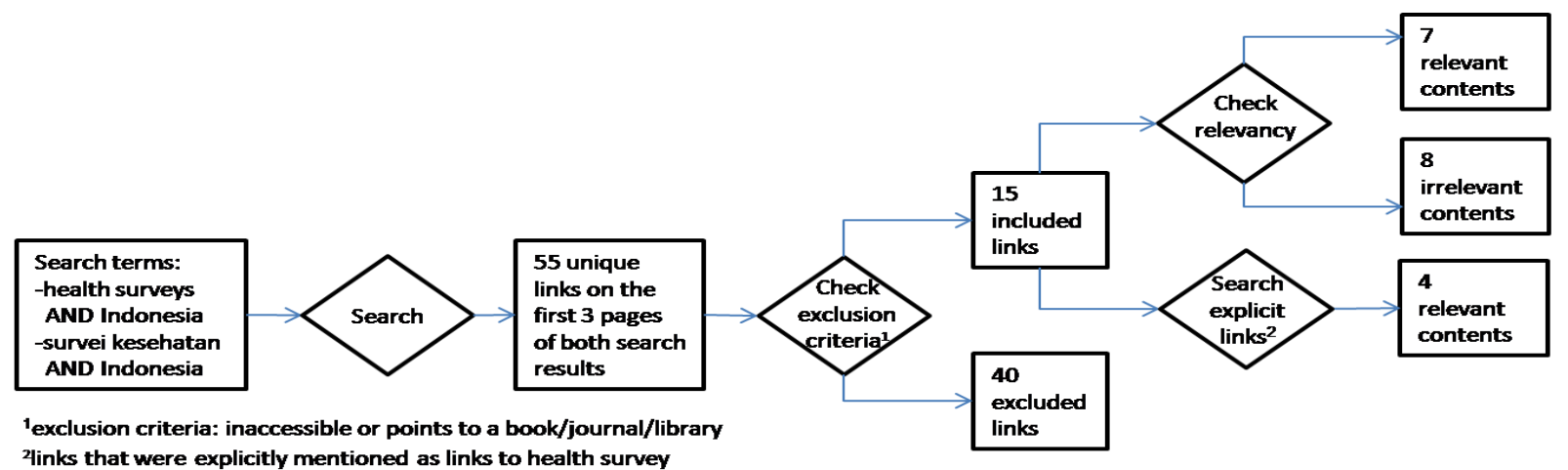

Figure 1-b. The diagram of search results using Google ${ }^{\mathrm{TM}}$ search engine

\section{2) Assessment of menu used to locate statistics on website}

Information on website was organized by menus. Users selected one of the menus on website homepage to get to the page displaying information of interest. In previous study by ORC Macro [[8]] various menus that lead to the page displaying health statistics were identified and classified into good menus and inappropriate/misleading menus. Good menus were those whose titles were intuitive enough for users to guess that they lead to the statistics of interest and that allowed for efficient search i.e. by providing search tool [[8]]. Menus titled "Health Statistics" and "Health Topics", as well as menu titled "Publications" that provided search tool for searching among various statistical and non-statistical reports were classified as good menus [[8]]. Meanwhile, inappropriate/ misleading menus were those whose titles were not intuitive and that required users to search more to find the statistics of interest [[8]]. Government agency was often divided into divisions/departments. Menu using a department's name as its title was classified as inappropriate/misleading as it was not intuitive and required users to know what department was responsible for disseminating statistics [[8]]. Menu titled "Publications" that did not provide search tool required users to search manually among a large number of reports and thus, was classified as inappropriate [[8]]. In this study, various menus used to locate statistics were described and classified into good and inappropriate/misleading menus.

\section{3) Assessment of mode of presentation and means of access to statistics}

It has been suggested that visual displays such as graph, chart and map are likely to be more effective in conveying information [[9]]. Additionally, the ability to query and request for specific statistics and to have access beyond reading them online, i.e. to export/print, may assist users in finding, extracting and using desired information [[8],[10],[11]]. In this study, 
we measured the proportion of sites using visual displays for presentation and providing feature to query/export/print statistics.

4) Assessment of available statistics

Available statistics were classified into statistics on mortality and cause of death, morbidity and health status, risk factors, service provision and health resources [[5]]. Examples of health statistics assessed were infant mortality rate, HIV prevalence, prevalence of obesity, antenatal care coverage, and number of midwives. Statistics from health surveys were further classified according to the administrative level at which the survey was conducted as well as the administrative level at which the statistics were reported on the web: national, regional, province, district and sub-district.

5) Proposal for a new dissemination website and its subsequent evaluation

Assessment results were used to derive design specification, and a prototype health statistics portal website was developed. Further, a preliminary usability test was conducted with 10 subjects, composed of health professionals and University of Tokyo's medical students from Indonesia, to measure how well users can use the portal site to find health statistics. This study was approved from the Ethics Committee of Graduate School of Medicine, the University of Tokyo (No.3125).

\section{Results}

Review of the characteristics of websites offering health statistics for Indonesia:

1) Identification of sources of health statistics available on the web

49 sources were identified on 18 governmental, 8 international and 5 non-government websites. They were classified into: census, vital registration, survey, estimation/projection/modeling, service/ surveillance and administrative records [[14]]. 31 sources $(63 \%)$ were found on government sites, and only 13 sources $(26 \%)$ and 17 sources $(35 \%)$ were found on non-government and international sites (Figure 2). 


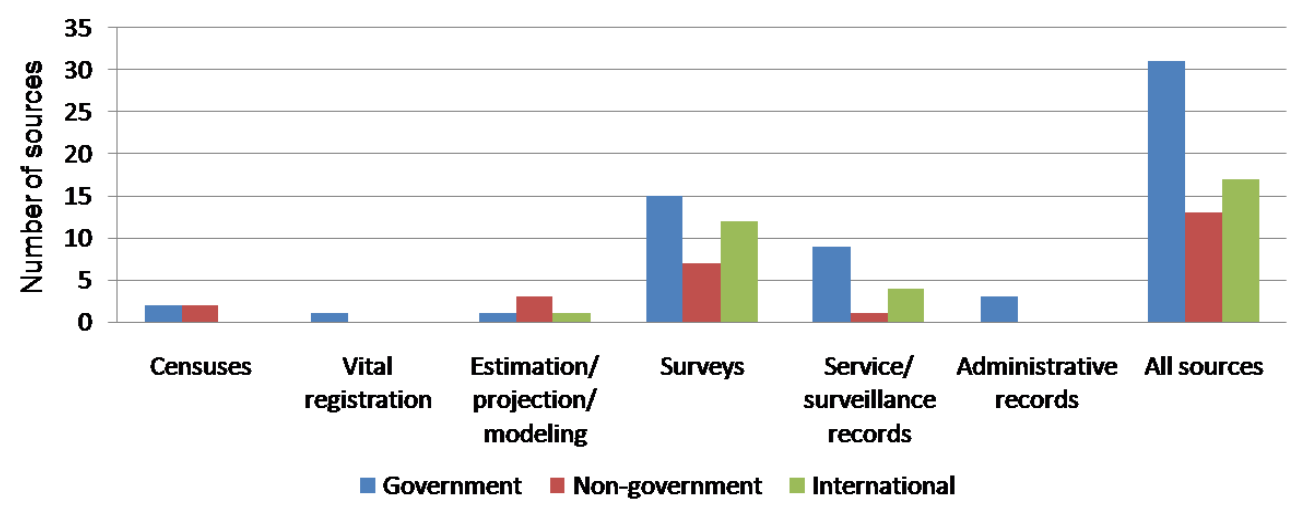

Figure 2. The difference of sources of public health statistics in the governmental, non-governmental and international websites

Of 31 sources on government sites, only 12 sources (39\%) were distributed to other site: 5 sources were distributed to non-government site, 5 sources were distributed to international site, and 2 sources were distributed to other government site.

\section{2) Assessment of menu used to locate statistics on website}

49 menus that lead to statistics were identified. Titles of the menus were "Health statistics", "Health topics", "News/Articles", "Download", and "Publications". Additionally, the name of a survey, data repository/database, country, or divisional part of the organization who owns the website was also often used as menu's title. In addition to menus titled "Health Statistics", "Health Topics" and "Publications" (with search tool), menus using the name of a survey, data repository/ database and country as their titles were classified as good menus.

Menus using the name of a survey/data repository/database were accompanied by explanation about the survey/data repository/ database and the statistics collected and thus, allowed users to recognize them as the menus to statistics. Organizing statistics by countries from which they came from, on the other hand, allowed users to search efficiently for data from a specific country. 15 of 17 menus (88\%) on international sites, 7 of 10 menus (70\%) on non-government sites, and 11 of 22 menus (50\%) on government sites were classified as good menus (Figure 3).

In addition to menus titled "Publications" (without search tool), menus titled "News/Articles" and "Download" were classified as inappropriate/misleading as they were not intuitive enough for users to guess that they lead to statistics and required more efforts to separate statistics from other (descriptive) information. 16 of 49 menus (33\%) were classified as inappropriate/misleading and 11 of them $(69 \%)$ were found on government websites. 
3) Assessment of mode of presentation and means of access to statistics

Of 31 websites, 26 websites $(84 \%)$ and 16 websites (52\%) used report and table to present statistics. Only 7 websites (23\%) and 12 websites (39\%) used map and graph/chart. Further, only 10 websites (32\%), 12 websites (39\%) and 6 websites (19\%) provided feature to query, export and print the statistics.

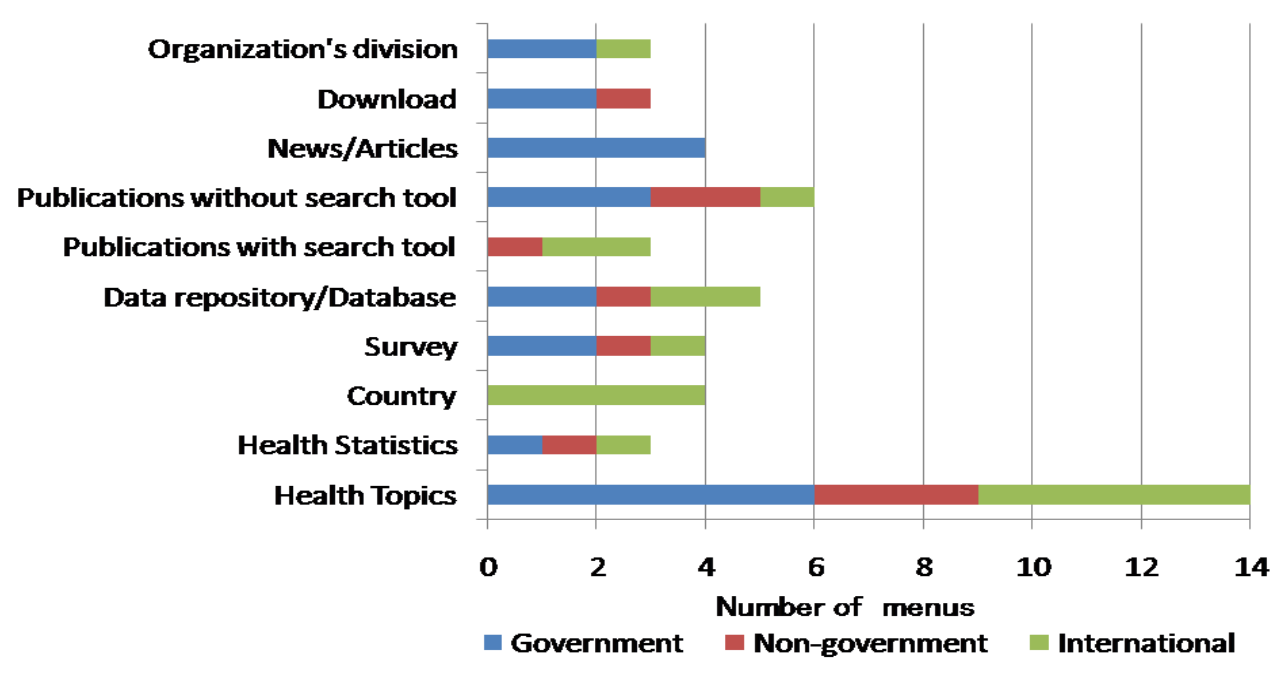

Figure 3. The characteristics of public health statistics related websites' menus in governmental, non-governmental and international websites

\section{4) Assessment of available statistics}

34 sources (69\%), 28 sources $(57 \%)$ and 25 sources $(51 \%)$ reported morbidity, risk factors and service provision statistics. Only 12 sources (24\%) and 18 sources (37\%) reported health resources and mortality statistics. 31 sources (63\%) were household surveys. 11 surveys $(35 \%)$ and 2 surveys $(6 \%)$ were conducted at district and sub-district level - the two lowest levels of administrative division in Indonesia. Only 8 surveys (26\%) were reported at district level and none was reported at sub-district level. Others (74\%) were reported at higher administrative levels.

5) Proposal for a new dissemination website and its subsequent evaluation

i) Design specification and development of statistics portal website

Based on the assessment results, there was low data accessibility. Internet users often faced difficulty in locating statistics on website. The menus used to locate statistics were often not intuitive enough for users to guess that they lead to statistics. Possible solutions include providing guidance on how to locate statistics on website and/or providing one access point that aggregates direct links to report, table, graph, chart, and map containing the statistics. 
Statistics portal site that offered both solutions was developed. The portal site was built using Joomla! ${ }^{\mathrm{TM}}$ content management system [[13]] and consisted of a homepage and 6 other web pages (URL: http://www.indopublichealth.comule.com). Each web page was dedicated for a health topic and divided into 4 sections. The topics were "Communicable diseases", "Non-communicable diseases", "Maternal \& Child health", "Lifestyle \& Environmental health", and "Service provision \& Health resources". They were chosen by identifying common themes shared by various statistics that we surveyed.

Figure 4 shows the portal site's user interface: (A) menus built from health topics, (B) "Dataset \& Statistics" section consisted of various link to statistics, (C) "Latest News" section consisted of public health-related news feeds, (D) "Search Websites" section consisted of Google custom search engine for searching on public health-related websites, and (E) "Resources" section consisted of various public health-related documents.

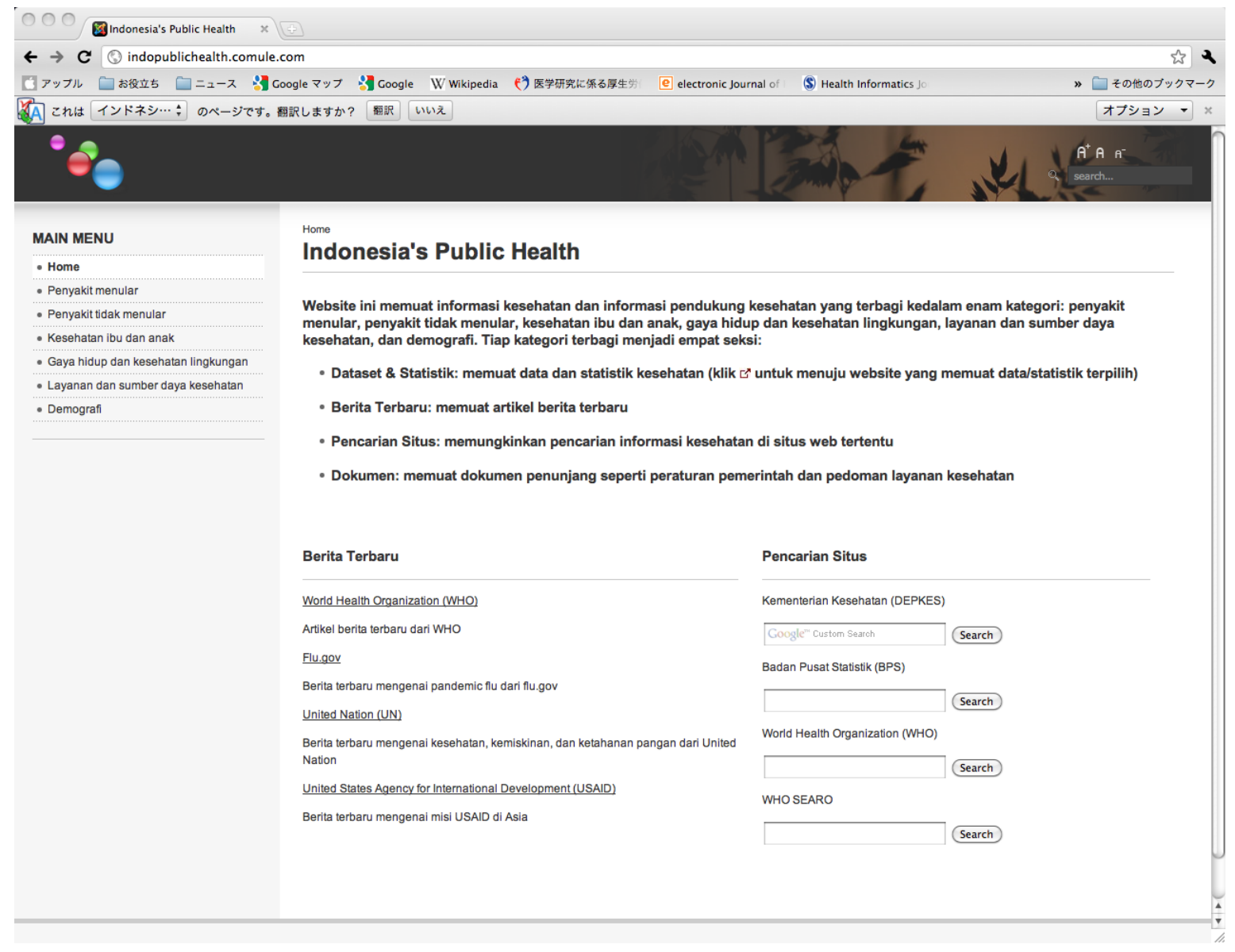

Figure 4. The graphical user interface of the developed portal site's based on our survey results. (http://indopublichealth.comule.com) 
ii) Preliminary usability test

A preliminary usability test was conducted with 10 subjects; for subject characteristics, see Table 1.

Table 1. The participants' characteristics in the usability test $(n=10)$

\begin{tabular}{|l|l|}
\hline Variable & $\mathrm{n}(\%)$ \\
\hline Sex & \\
\hline Male & $4(40)$ \\
\hline Female & $6(60)$ \\
\hline Age & \\
\hline Means \pm SD & $38.7 \pm 2.21$ \\
\hline Occupation & \\
\hline Student & $5(50)$ \\
\hline Health professional & $5(50)$ \\
\hline Education & \\
\hline Undergraduate & $5(50)$ \\
\hline Graduate & $5(50)$ \\
\hline
\end{tabular}

Subjects were given 3 questions. Each question asked them to search for health statistics using the portal site (a total of 30 searches; 3 for each subject). Performance data - score and time spent for each question were collected. Additionally, a questionnaire was used to collect demographic data and users' subjective ratings [[14]]. Most subjects earned high scores indicating that their success rate was quite high (Figure 5). Subjects reported being somewhat satisfied or very satisfied with the result obtained from $87 \%$ of the searches. The average time spent to find particular statistics was 1 minute and 58 seconds (Mean=118.4sec [SD = 33.7]) (Figure 5).

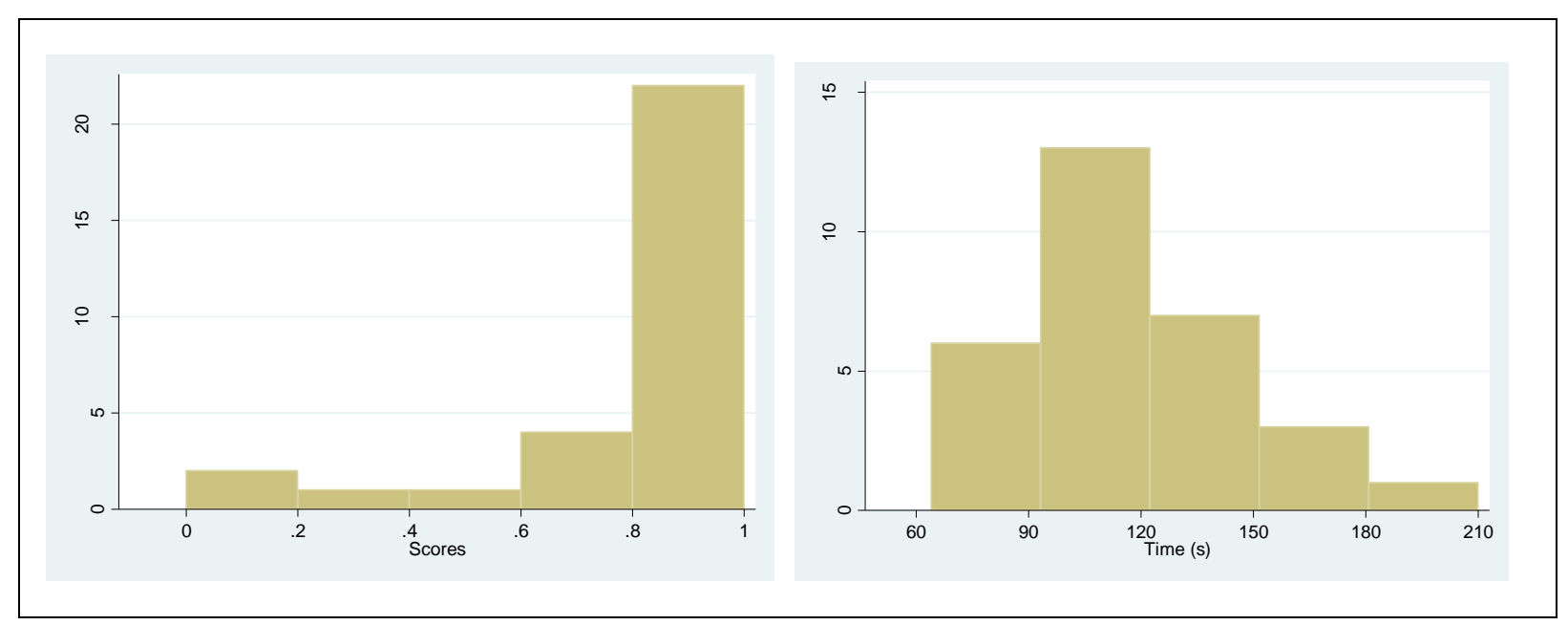

Figure 5. The distribution of search scores (left) and time (right) 
Subjects gave neutral to favorable ratings on satisfaction with the time spent on $63 \%$ of the searches. Subjects also gave neutral to favorable ratings for website usefulness on all searches, and for website ease of use on $97 \%$ of the searches (Figure 6). Further, subjects gave neutral to favorable ratings on frustration experience on $53 \%$ of the searches (Figure 6).

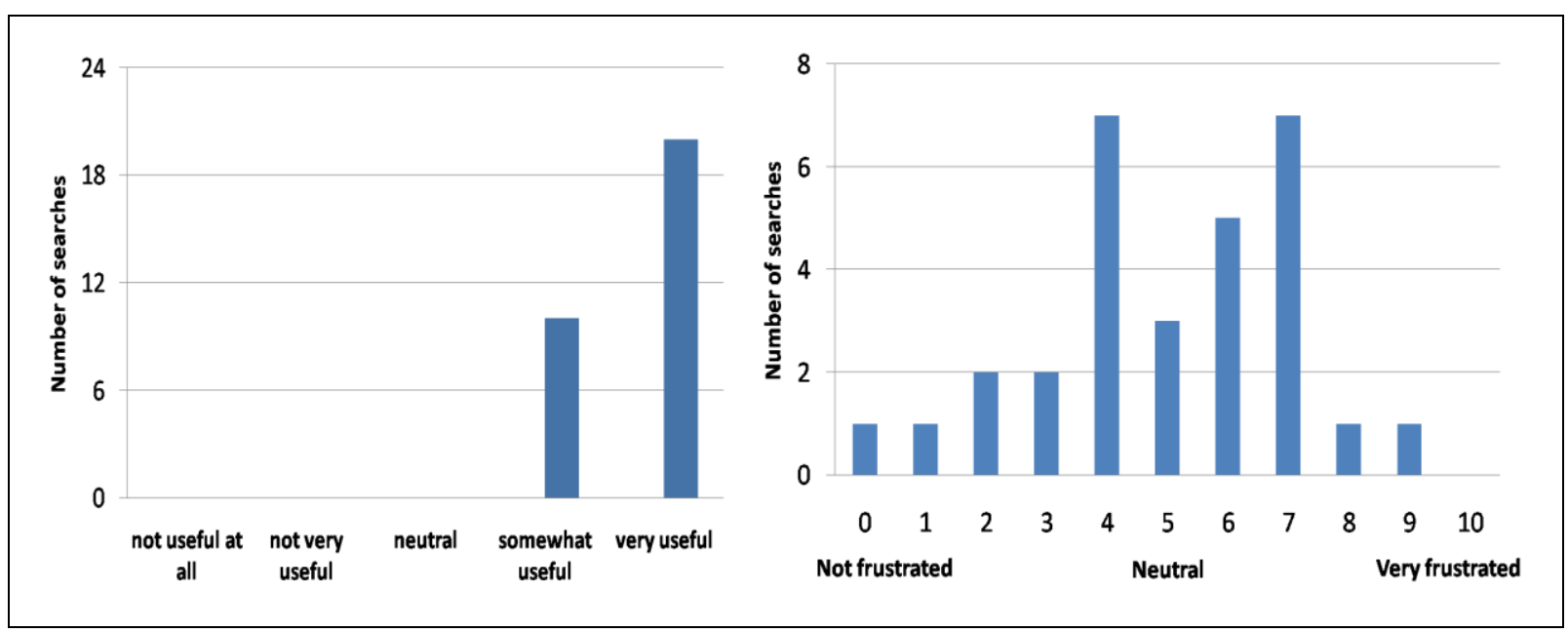

Figure 6. Portal site usefulness (left) and level of frustration (right)

The frustration was, however, not caused by the portal site but by the difficulties found on the websites that provided the statistics, and those caused by subjects' mistakes i.e. mistake in not thoroughly reading the questions. Minor usability issues pointed out by subjects included those related to the unavailability of language options and advance search feature.

\section{Discussion}

Various sources of health statistics have been identified including census, vital registration and survey. Most were on government websites. The availability of various sources on and, for some sources, only on these sites emphasizes the role of Government as the main producer and distributor of information. The roles of international and non-governmental organizations, meanwhile, were limited to particular sources only. Despite of its role, government's statistics were seldom shared by multiple sites. The availability of information on multiple sites could increase the chance of finding that information. The easier to find the information is, the more useful it is for its users [[8]]. Thus, for existing statistics to be more useful, they should be distributed to as many sites as possible.

To get to the page displaying health statistics, user needs to select one of the menus on website homepage. The titles of the menus especially on government websites were, however, often not intuitive enough for users to guess that they lead to statistics. In addition to be 
misguided, they were often required to search manually among a large number of information. The more difficult to find the information is, the less useful it is for its users [[8]]. Thus, appropriate title should be used for menu and, to allow for efficient search, either a search tool was provided or the statistics were stored separately from other information.

Despite of the suggestion that visual displays such as graph, chart and map are likely to be more effective in conveying information [[9]], they were infrequently used by the websites surveyed here. Additionally, means of access to statistics was limited as users were often unable to query them and could only access them online. Due to these reasons, intended information may not reach audiences and consequently, it is not used. Addressing these issues could potentially lead to an increased use of information in public health practice.

As Internet users worldwide continue to increase [[15]], the role of the Internet as dissemination tool becomes more significant. Information, especially those come from reliable sources such as the Government, should be reported on the web. Our findings showed that various statistics have been reported on the web. Those on mortality and health resources were, however, particularly difficult to find. Government and other responsible organizations should be aware of this, and act accordingly to address this information gap.

Surveys conducted at district/sub-district level should be able to provide detailed statistics on the web. Unfortunately, because some were reported at higher administrative levels, the availability of detailed statistics on the web was less than expected. For the information on the web to be useful, especially to local government responsible for the implementation of health program at district or sub-district level, it should be reported in detail enough to be used in practice. Reporting statistics at the same level at which the data were collected is one way to achieve this purpose.

Internet users often faced difficulty in locating statistics on website. The menus used to locate statistics were often not intuitive enough for users to guess that they lead to statistics. To improve data accessibility, statistics portal site was developed. Usability test of the portal site demonstrated its potential to assist users in locating statistics and provided us with necessary information to further improve its usability.

Despite of all efforts that have been taken to make statistics available on the web, information gap still exists and additionally, existing information may not be as useful as it was intended to be. Related to information gap, attention should be brought to 2 issues: the scarce availability of health resources and mortality statistics, and the less-than-expected availability of sub-district/district level statistics. Appropriate actions should be taken in order to address 
these issues. Further, there are several reasons why existing information may not be very useful: a) it is disseminated only on limited websites, b) it is difficult to locate, especially those on government websites, and c) it is not effectively communicated. If the information is to be used as basis for action, users should not have difficulty in finding and using it i.e. they should have access whenever they need to and be able to extract the information without any difficulty.

Health statistics are reported on the web with the intent of making them useful. We have performed, to the best of our knowledge, the first research that attempted to describe health statistics dissemination efforts for Indonesia. This has enabled us to highlight significant issues and suggest potential ways for improvement. Although we may have missed some information, due to imperfect search strategy, it is unlikely that this will lead to a different message than the one conveyed here. The bottom line, actions are needed to ensure the usability of health statistics on the web. As one of such actions, we developed statistics portal website to improve the currently low data accessibility. Usability test of the portal site showed promising results but subsequent evaluation with more and a wider range of subjects would be needed to further improve its usability.

\section{Disclosure}

The authors have no personal financial or institutional interest in any of the drugs, materials, or devices described in this article.

\section{Acknowledgements}

The author would also like to thank all of the subjects for their generous participation in this study.

\section{Corresponding author:}

Hiroshi Oyama, M.D.

Department of Clinical Information Engineering,

Health Services Science Division, School of Public Health,

Graduate School of Medicine, The University of Tokyo.

Address:

7-3-1 Hongo, Bunkyo-ku, Tokyo, 113-8655, Japan.

Tel\&Fax: +81-3-5841-1893

E-mail: hoyama-nsu@umin.ac.jp 


\section{References}

[1]The World Bank. Indonesia policy briefs - ideas for the future: improving Indonesia's health outcomes. Jakarta: The World Bank; 2005.

[2]The World Bank. Indonesia's doctors, midwives and nurses: current stock, increasing needs, future challenges and options. Jakarta: The World Bank; 2009.

[3]Institute of Medicine. The future of public health. Washington, D.C.: National Academy Press; 1988.

[4]Pappaioanou M, Malison M, Wilkins K, Otto B, Goodman RA, Churchill RE, White M, Thacker B. Strengthening capacity in developing countries for evidence-based public health: the data for decision-making project. Social Science \& Medicine 2003; 57: 1925-1937.

[5]Boerma JT, Stansfield SK. Health statistics now: are we making the right investments? Lancet 2007; 369: 779-786.

[6]World Health Organization Regional Office for South-East Asia (WHO SEARO). Indonesia country health profile - development of the health system. WHO SEARO; 2007. http://www.searo.who.int/ en/Section313/Section1520_6822.htm. Accessed January 1, 2010.

[7]Raban MZ, Dandona R, Dandona L. Essential health information available for India in the public domain on the Internet. BMC Public Health 2009; 9: 208. doi: 10.1186/1471-2458-9-208.

[8]ORC Macro, Inc. Evaluation of the state-based integrated health information system. Contract \# 200-96-0598. Task Order No 23. Atlanta, Georgia: ORC Macro, Inc; 2000. http://sbh.orcmacro.com/ RevisedEvalof-StateFinalReport.pdf. Accessed February 8, 2010.

[9]Goodman RA, Patrick LR, Howard RJ. Communicating information for action within the public health system. In: Teutsch SM, Churchill RE, eds. Principles and practice of public health surveillance. 2nd ed. New York: Oxford University Press; 2000: 168-175.

[10]U.S. Department of Health and Human Services. Research-based web design \& usability guidelines. Washington, D.C.: U.S. Department of Health and Human Services. http://www.usability.gov/guidelines/guidelines_book.pdf. Accessed March 15, 2010.

[11]ORC Macro, Inc. A guide for public health agencies developing, adopting, or purchasing interactive web-based data dissemination systems. Contract \# 200-96-0598. Task Order No 23. Atlanta, Georgia: ORC Macro, Inc. http://www.cdc.gov/epo/dphsi/files/WDDSGuideF3.doc. Accessed April 23, 2010.

[12]AbouZahr C, Boerma T. Health information systems: the foundation of public health. Bulletin of the World Health Organization 2005; 838: 578-583 
[13]Joomla! ${ }^{\mathrm{TM}}$. Joomla content management system. http://www.joomla.org. Accessed May 1, 2010.

[14]Ceaparu I. Finding government statistical data on the web: a case study of FedStats. IT \& Society 2003; 13: 1-17.

[15]Internet World Stats. Usage and population statistics. Internet usage statistics. World Internet users and population stats. Internet World Stats; 2010. http://www.internetworldstats.com/stats.htm. Accessed May 30, 2010. 